Bull. Austral. Math. Soc.

VOL. 51 (1995) [153-162]

\title{
APPROXIMATE CONVERSION OF BÉZIER CURVES
}

\author{
Yungeom Park, U Jin Chol and Ha-Jine KImN
}

\begin{abstract}
The methods for generating a polynomial Bézier approximation of degree $n-1$ to an $\boldsymbol{n}$ th degree Bézier curve, and error analysis, are presented. The methods are based on observations of the geometric properties of Bézier curves. The approximation agrees at the two endpoints up to a preselected smoothness order. The methods allow a detailed error analysis, providing a priori bounds of the pointwise approximation error. The error analysis for other authors' methods is also presented.
\end{abstract}

\section{INTRODUCTION}

The process for generating a polynomial Bézier approximation of degree $n-1$ to an $n$th degree Bézier curve is called degree reduction. In general degree reduction of Bézier curves addresses the following problem.

Problem 1. Let $\left\{b_{i}\right\}_{i=0}^{n} \subset R^{*}$ be a given set of (control) points which define the Bézier curve

$$
b^{n}(t)=\sum_{i=0}^{n} b_{i} B_{i}^{n}(t), \quad 0 \leqslant t \leqslant 1
$$

in terms of Bernstein polynomials $B_{i}^{n}(t)=\left(\begin{array}{c}n \\ i\end{array}\right)(1-t)^{n-i} t^{i}$ of degree $n$. Then find another point set $\left\{q_{i}\right\}_{i=0}^{m} \subset R^{\prime}$ defining the (approximating) Bézier curve

$$
q^{m}(t)=\sum_{i=0}^{m} q_{i} B_{i}^{m}(t), \quad 0 \leqslant t \leqslant 1
$$

of lower degree $m<n$ so that a suitable distance function $d\left(b^{n}, q^{m}\right)$ between $b^{n}$ and $q^{m}$ is minimised on the interval $[0,1]$.

The necessity to determine the degree reduced curve by approximation is manifest, since degree reduction is not exactly possible in general, in contrast to the reverse question of degree elevation. In so doing, the degree reduction can be accomplished in a number of ways. Forrest [6] proposed a geometrical algorithm preserving the tangent

Received 4th May, 1994

This work was partially supported by KOSEF under the grant number 91-08-00-01.

Copyright Clearance Centre, Inc. Serial-fee code: 0004-9729/95 \$A2.00+0.00. 
at two endpoints. Farin's degree reduction scheme [4] is compared with the rational Bézier curves case. Forrest's and Farin's method are presented without error analysis. Watkins and Worsey [9] and Lachance [7] suggested that Chebyshev polynomials be used in the degree reduction process. Eck [2] generalised Forrest's method by using constrained Chebyshev polynomials.

Now, the approximation $q^{m}$ depends critically on the chosen distance or error function $d\left(b^{n}, q^{m}\right)$ to be minimised. Here, the most appropriate metric in geometrical terms would be the Hausdorff distance; see Degen [1] for a detailed discussion. It measures a special kind of geometrical distance between the two compact sets $\left\{b^{n}(t): t \in[0,1]\right\}$ and $\left\{q^{m}(t): t \in[0,1]\right\}$ by ignoring the respective parametrisations. The consequent approximation process is explained in Degen [1] and Eisele [3], where nonlinear theory is used to determine the curve $q^{m}$ numerically.

On the other hand, we are always interested in explicit formulas for the approximation $\boldsymbol{q}^{\boldsymbol{m}}$. Therefore, we shall use the much simpler distance function

$$
d\left(b^{n}, q^{m}\right)=\max \left\{\left\|b^{n}(t)-q^{m}(t)\right\|: t \in[0,1]\right\}
$$

which measures the maximal distance with respect to the parametrisation. An immediate consequence of this error metric $d\left(b^{n}, q^{m}\right)$ is that the geometry of $b^{n}$ is approximated as well as its parametrisation.

The main advantage of using (1.1) is the decomposition of the original Problem 1 into $s$ subproblems. Each subproblem consists of minimising the componentwise error function

$$
d\left(b_{j}^{n}, q_{j}^{m}\right)=\max \left\{\left|b_{j}^{n}(t)-q_{j}^{m}(t)\right|: t \in[0,1]\right\}, \quad(j=1, \ldots, s)
$$

where we introduce the notation $f(t)=\left(f_{1}(t), \ldots, f_{d}(t)\right)^{T}$. Then the minimal distance $(1.1)$ is determined by $d\left(b^{n}, q^{m}\right)=\left(\sum_{j=1}^{\infty} d\left(b_{j}^{n}, q_{j}^{m}\right)^{2}\right)^{1 / 2}$.

Hence, it suffices from now on to investigate the single-valued or functional case only. Furthermore, we shall restrict ourselves to the very special case $m=n-1$ since explicit solutions are known only for this exceptional case. This is carried out in the next section where the following reformulated problem is solved.

PROBLEM 2. Let $\left\{b_{i}\right\}_{i=0}^{n}$ be a given set of real coefficients which define the Bézier function

$$
b^{n}(t)=\sum_{i=0}^{n} b_{i} B_{i}^{n}(t), \quad 0 \leqslant t \leqslant 1 .
$$

Then find another set of real coefficients $\left\{q_{i}\right\}_{i=0}^{n-1}$ defining the approximation

$$
q^{n-1}(t)=\sum_{i=0}^{n-1} q_{i} B_{i}^{m}(t), \quad 0 \leqslant t \leqslant 1
$$


by minimising the uniform error function $d\left(b^{n}, q^{n-1}\right)=\max \left\{\left|b^{n}(t)-q^{n-1}(t)\right|\right\}$ on the interval $[0,1]$.

In the second section of the paper, the methods for degree reduction are presented with an investigation of the geometric property of Bézier curves that was needed to develop the scheme. Section 3 then discusses how to extend this construction so that boundary constraints can be involved. The authors aim to develop a geometric method for generating lower degree approximations which, in the uniform norm, are within some preset error tolerance of the prescribed Bézier curve. This means that the scheme for approximation must be combined with a subdivision algorithm. This question is also addressed in the third section. The techniques used in the approximation algorithms admit a detailed error analysis for the other authors' methods. The error analyses for the methods developed by Forrest [6], Farin [4] and Eck [2] are presented in Section 4.

\section{APPROXIMATION}

Let us consider first the reverse problem. For given Bézier curve $q^{n-1}$ with $n$ coefficients $q_{0}, q_{1}, \ldots, q_{n-1}$, one might want to add another coefficient yet leave the shape of the curve unchanged - this corresponds to raising the degree of the Bézier curve by one (this process is called degree elevation) The new coefficients $b_{i}$ must satisfy (see Farin [5])

$$
b_{i}=\frac{i}{n} q_{i-1}+\frac{n-i}{n} q_{i}, \quad(i=0, \ldots, n)
$$

Equation (2.1) can be used to derive two different recursive extrapolation formulas for the generation of the coefficients $q_{i}$ from the $\left\{b_{i}\right\}$ :

$$
q_{0}^{l}=b_{0}, \quad q_{i}^{l}=\frac{n}{n-i} b_{i}-\frac{i}{n-i} q_{i-1}^{l}, \quad(i=1, \ldots, n-1)
$$

or

$$
q_{n-1}^{r}=b_{n}, \quad q_{i-1}^{r}=\frac{n}{i} b_{i}-\frac{n-i}{i} q_{i}^{r}, \quad(i=n-1, \ldots, 1) .
$$

If $\frac{d^{n}}{d t^{n}} b^{n}(t) \equiv 0$ or equivalently $\Delta^{n} b_{0}=0$ where the $r$ th forward difference of the coefficients is defined explicitly by

$$
\Delta^{r} b_{i}=\sum_{j=0}^{r}\left(\begin{array}{l}
r \\
j
\end{array}\right)(-1)^{r+j} b_{i+j},
$$

then $b^{n}$ is an artificially degree elevated function and we have $q_{i}^{l}=q_{i}^{r}$ for all $i$ meaning that the degree reduction scheme reproduces the original function exactly. 
For a function $b^{n}$ with $\Delta^{n} b_{0} \neq 0$, let $V=\left|\Delta^{n} b_{0}\right|=\sum_{j=0}^{n}(-1)^{j}\left(\begin{array}{c}n \\ j\end{array}\right) b_{j}$. Then

$$
\sum_{i=0}^{n}(-1)^{i}\left(\begin{array}{c}
n \\
i
\end{array}\right) \widehat{b}_{i}=0
$$

where

$$
\widehat{b}_{i}=b_{i}-\frac{(-1)^{i}}{\left(\begin{array}{c}
n \\
i
\end{array}\right)} \alpha_{i} V
$$

and $\sum_{i=0}^{n} \alpha_{i}=1$. The function $\widehat{b}^{n}(t)$ with coefficients $\widehat{b}_{i}(i=0, \ldots, n)$ is an artificially degree elevated function. Now, in order to be able to construct approximations we introduce additional degrees of freedom, namely real weighting factors $\alpha_{i}$. The function $\widehat{q}^{n-1}(t)$ with coefficients $\widehat{q}_{i}(i=0, \ldots, n-1)$ which are obtained from $(2.2)$ or $(2.3)$ is degree reduced.

Since the functions $\widehat{b}^{n}(t)$ and $\widehat{q}^{n-1}(t)$ are the same and we set $\widehat{q}^{n-1}(t)=q^{n-1}(t)$, the approximation error is

$$
d\left(b^{n}, q^{n-1}\right) \leqslant \max |E(t)| \cdot|V|,
$$

where

$$
E(t)=\sum_{i=0}^{n} \gamma_{i} B_{i}^{n}(t)
$$

with $\gamma_{i}=(-1)^{i} \alpha_{i} /\left(\begin{array}{c}n \\ i\end{array}\right)$.

In order to obtain the factors $\alpha_{i}$ which minimise the error we first consider the Chebyshev polynomial $T_{n}(x)$ of degree $n$ which may be written as

$$
T_{n}(x)=\cos (n \cdot \arccos (x)), \quad x \in[-1,1] .
$$

Alternatively, it can be computed in monomial form by a simple recurrence formula. In contrast to that the following representation in Bernstein form is not so familiar (see Eck [2]):

$$
T_{n}(2 t-1)=\sum_{i=0}^{n}(-1)^{n+i} \frac{\left(\begin{array}{c}
2 n \\
2 i
\end{array}\right)}{\left(\begin{array}{c}
n \\
i
\end{array}\right)} B_{i}^{n}(t), \quad t \in[0,1] .
$$

Now, we set $E(t)=T_{n}(2 t-1)$; and then we find the factors $\alpha_{i}$ by

$$
\alpha_{i}=\frac{1}{2^{2 n-1}}\left(\begin{array}{c}
2 n \\
2 i
\end{array}\right), \quad(i=1, \ldots, n)
$$


such that $\sum_{i=0}^{n} \alpha_{i}=1$. Since $\max \left\{\left|T_{n}(x)\right|: x \in[-1,1]\right\}=1$, the approximation error is

$$
d\left(b^{n}, q^{n-1}\right) \leqslant \frac{1}{2^{2 n-1}}|V|
$$

For convenience of computation of (2.5) we choose the factors $\alpha_{i}$ by

$$
\alpha_{i}=\frac{1}{2^{n}}\left(\begin{array}{l}
n \\
i
\end{array}\right), \quad(i=1, \ldots, n)
$$

such that $\sum_{i=0}^{n} \alpha_{i}=1$. Then the approximation error is

$$
d\left(b^{n}, q^{n-1}\right) \leqslant \frac{1}{2^{n}}|V|
$$

\section{Approximation with Smoothness}

The function $b^{n}$ and the approximation $\widehat{q}^{n-1}$ do not coincide in general at the two boundaries. We introduce new $n$ factors $\beta_{i}(i=0, \ldots, n-1)$ in order to overcome this problem. The function $q^{n-1}(t)$ defined by the coefficients

$$
q_{i}=\widehat{q}_{i}+\beta_{i} V, \quad(i=0, \ldots, n-1)
$$

is also an approximation for the function $b^{n}$. In Watkins and Worsey's work [9], $C^{0}$ continuity at the endpoints is obtained by changing the first and last coefficient of $\hat{q}^{n-1}$ so that interpolation holds. In our case, this procedure corresponds to simply defining $\beta_{0}=\alpha_{0}$ and $\beta_{n-1}=(-1)^{n} \alpha_{n}$, whereas the remaining factors are zero.

Generally, to solve Problem 2 with the additional constraints

$$
\left.\frac{d^{r}}{d t^{r}} b^{n}(t)\right|_{t=t_{0}}=\left.\frac{d^{r}}{d t^{r}} q^{n-1}(t)\right|_{t=t_{0}} \quad(r=0, \ldots, \theta), \quad 0 \leqslant \theta \leqslant \frac{n-1}{2}
$$

for $t_{0}=0$ and $t_{0}=1$, we obtain the factors $\beta_{i}$

$$
\beta_{0}=\gamma_{0}, \quad \beta_{i}=\frac{n}{n-i} \gamma_{i}-\frac{i}{n-i} \beta_{i-1}, \quad(i=1, \ldots, \theta)
$$

and

$$
\beta_{n-1}=\gamma_{n}, \quad \beta_{i-1}=\frac{n}{i} \gamma_{i}-\frac{n-i}{i} \beta_{i}, \quad(i=n-1, \ldots, n-\theta)
$$

by using the forward difference formula (2.4). Equations (3.1) and (3.2) are rewritten as

$$
\beta_{i}=\frac{(-1)^{i}}{\left(\begin{array}{c}
n-1 \\
i
\end{array}\right)} \sum_{j}^{i}(-1)^{j}\left(\begin{array}{l}
n \\
j
\end{array}\right) \gamma_{j}, \quad(i=1, \ldots, \theta)
$$


and

$$
\beta_{i}=\frac{(-1)^{i+1}}{\left(\begin{array}{c}
n-1 \\
i
\end{array}\right)} \sum_{j=i+1}^{n}(-1)^{j}\left(\begin{array}{l}
n \\
j
\end{array}\right) \gamma_{j}, \quad(i=n-1, \ldots, n-\theta)
$$

Since the functions $\widehat{b}^{n}(t)$ and $\widehat{q}^{n-1}(t)$ are the same and

$$
B_{i}^{n-1}(t)=\frac{n-i}{n} B_{i}^{n}(t)+\frac{i+1}{n} B_{i+1}^{n}(t),
$$

the approximation error is

$$
d\left(b^{n}, q^{n-1}\right) \leqslant \max \left|E_{d}(t)\right| \cdot|V|
$$

where

$$
E_{s}(t)=\sum_{i=0}^{n}\left\{\gamma_{i}+\frac{n-i}{n} \beta_{i}+\frac{i}{n} \beta_{i-1}\right\} B_{i}^{n}(t) .
$$

In order to obtain the relation $E_{s}(t)$ and $E(t)$ in (2.6), we use equations (3.3) and (3.4). Hence

$$
E_{s}(t)=E(t)+2 \sum_{i=0}^{\theta} \gamma_{i} B_{i}^{n}(t)+2 \sum_{i=n-\theta}^{n} \gamma_{i} B_{i}^{n}(t)
$$

If the factors $\left|\gamma_{i}\right|$ have a symmetric property, that is $\left|\gamma_{i}\right|=\left|\gamma_{n-i}\right|$ for $i=0,1, \ldots, n$, we have

$$
\max \left|E_{s}(t)\right|=\max |E(t)|+4(\theta+1) \max _{0 \leqslant i \leqslant \theta}\left|\gamma_{i}\right|
$$

In the case that the $\alpha_{i}$ are chosen as in (2.7), the approximation error is

$$
d\left(b^{n}, q^{n-1}\right) \leqslant \frac{1}{2^{2 n-1}}(1+C)|V|
$$

where $C=4\left(\begin{array}{c}2 \pi \\ 2 \theta\end{array}\right)\left(\begin{array}{c}n \\ \theta\end{array}\right)^{-1}$.

Also, in the case that the $\alpha_{i}$ are chosen as in (2.8), the approximation error is

$$
d\left(b^{n}, q^{n-1}\right) \leqslant \frac{4 \theta+5}{2^{n}}|V|
$$

The a priori estimated error (2.6) and (3.5) are useful together with the following observation: if we subdivide a function $b^{n}$ of degree $n k$-fold at equidistant parameter values $t_{i}=(i /(k+1))(i=1, \ldots, k)$, then the $n$th forward difference of the coefficients in each of the $k+1$ segments arising is $1 /(k+1)^{n}$ times the original difference $\Delta^{n} b_{0}$ of the function $b^{n}$. 
Therefore, to satisfy a preset tolerance $\varepsilon$ we have firstly to subdivide $b^{n}(t) k$-fold at $t_{i}$ and afterwards to degree reduce each of the $k+1$ pieces. The number $k$ of subdivisions is calculated immediately from (2.6) and (3.5) by

$$
k=\left\lfloor\left(\frac{C \cdot|V|}{\varepsilon}\right)^{1 / n}\right\rfloor
$$

where $\lfloor\cdot\rfloor$ denotes the floor function and the constant $C$ is $\max |E(t)|$ or $\max \left|E_{s}(t)\right|$. Obviously, $k=0$ means that no subdivision is necessary to satisfy the error tolerance.

\section{ERROR ANALYSIS FOR OTHER METHODS}

In this section, error analyses for other authors' methods are presented. The error as in Farin [4] and Forrest's [6] methods are computed. A part of Eck's [2] method is also considered.

We consider first the degree reduction method of Forrest. Both formulas (2.2) and (2.3) only represent approximations and one observes immediately that (2.2) produces reasonable approximations near to $b_{0}$ and (2.3) behaves decently near $b_{n}$. Therefore, Forrest [6] proposed combining both formulas by taking the left half of the coefficients from (2.2) and right half of the coefficients from (2.3), so

$$
\begin{aligned}
& q_{i}=q_{i}^{l}, \quad(i=0,1, \ldots), \\
& q_{i}=q_{i}^{r}, \quad(i=n-1, n-2, \ldots) .
\end{aligned}
$$

For odd $n$, the midpoint appearing is defined by $q_{i}=\left(q_{i}^{l}+q_{i}^{r}\right) / 2$ with $i=(n-1) / 2$.

The coefficients $((n-i) / n) q_{i}+(i / n) q_{i-1}$ of the artificially degree elevated function $q^{n-1}$ are obtained from

$$
b_{i}-\frac{(-1)^{i}}{\left(\begin{array}{c}
n \\
i
\end{array}\right)} V, \quad i=\frac{n}{2} \quad(n \text { even })
$$

and

$$
b_{i}-\frac{(-1)^{i}}{2\left(\begin{array}{c}
n \\
i
\end{array}\right)} V, \quad i=\frac{n-1}{2}, \frac{n+1}{2} \quad(n \text { odd })
$$

otherwise $b_{i}$ where the coefficients $q_{-1}$ and $q_{n}$ are negligible.

Thus we have for even $n$,

$$
b^{n}(t)-q^{n-1}(t)=\frac{(-1)^{n / 2}}{\left(\begin{array}{l}
n \\
\frac{n}{2}
\end{array}\right)} V B_{n / 2}^{n}(t)
$$


and for odd $n$,

$$
b^{n}(t)-q^{n-1}(t)=\frac{(-1)^{(n-1 / 2)}}{2\left(\begin{array}{c}
n \\
\frac{n-1}{2}
\end{array}\right)} V\left\{B_{(n-1) / 2}^{n}(t)-B_{(n+1) / 2}^{n}(t)\right\} .
$$

By comparison of these formulas with the scheme presented in the previous section, we have the factor $\alpha_{n / 2}=1$ for even $n$ and $\alpha_{(n-1) / 2}=\alpha_{(n+1) / 2}=1 / 2$ for odd $n$; otherwise $\alpha_{i}=0$ and the all $\beta_{i}$ 's equal zero.

For computation of error bounds, we substitute these factors into (3.5). Since the Bernstein polynomial $B_{i}^{n}$ has only one maximum and attains it at $t=i / n$,

$$
d\left(b^{n}, q^{n-1}\right) \leqslant \begin{cases}\left(\frac{1}{2}\right)^{n}|V| & n \text { is even } \\ \frac{1}{2 \sqrt{n}}\left(\frac{1}{4}-\frac{1}{4 n}\right)^{(n-1) / 2}|V| & n \text { is odd. }\end{cases}
$$

Some different error bounds for Forrest's method were explained in full detail in Park and Choi [8].

In order to be able to construct an approximation, Farin [4] extended Forrest's construction by introducing a weighted average of the form

$$
q_{i}=\left(1-\frac{i}{n-1}\right) q_{i}^{l}+\frac{i}{n-1} q_{i}^{r}, \quad(i=0, \ldots, n-1) .
$$

In Farin's method, the approximation is defined by

$$
q^{n-1}(t)=\sum_{i=0}^{n-1}\left\{\frac{n-1-i}{n-1} q_{i}^{l}+\frac{i}{n-1} q_{i}^{r}\right\} B_{i}^{n-1}(t)
$$

We obtain by solving the recurrence relation (2.2) and (2.3) that

$$
q^{n-1}(t)=\sum_{i=0}^{n-1}\left\{V_{i}-\frac{i}{n-1} V\right\} \frac{(-1)^{i}}{\left(\begin{array}{c}
n-1 \\
i
\end{array}\right)} B_{i}^{n-1}(t),
$$

where $V_{i}=\sum_{j=0}^{i}(-1)^{j}\left(\begin{array}{l}i \\ j\end{array}\right) b_{j}$.

If we artificially elevate the degree of $q^{n-1}$, then

$$
q^{n-1}(t)=\sum_{i=0}^{n}\left\{b_{i}-\frac{(-1)^{i} V}{(n-1)\left(\begin{array}{c}
n \\
i
\end{array}\right)}\right\} B_{i}^{n}(t) .
$$


Thus, we have

$$
b^{n}(t)-q^{n-1}(t)=\frac{V}{n-1} \sum_{i=0}^{n} \frac{(-1)^{i}}{\left(\begin{array}{l}
n \\
i
\end{array}\right)} B_{i}^{n}(t)
$$

and the factor $\alpha_{0}=\alpha_{n}=0, \alpha_{i}=1 /(n-1)$ for $i=1, \ldots, n-1$ and the all $\beta_{i}$ 's equal zero. The error bound is

$$
d\left(b^{n}, q^{n-1}\right) \leqslant \frac{1}{n-1}|V| .
$$

Eck [2] extended Farin's method by introducing additional degrees of freedom, namely real weighting factors $\lambda_{i}$ which blend the coefficients $q_{i}^{r}$ and $q_{i}^{l}$ together. In more detail, to define the new coefficients $q_{i}$, the following linear interpolation formula is used:

$$
q_{i}=\left(1-\lambda_{i}\right) q_{i}^{l}+\lambda_{i} q_{i}^{r}, \quad(i=0, \ldots, n-1)
$$

where

$$
\lambda_{i}=\frac{1}{2^{2 n-1}} \sum_{j=0}^{i}\left(\begin{array}{l}
2 n \\
2 j
\end{array}\right), \quad(i=0, \ldots, n-1) .
$$

Then the approximation error is (see Eck [2, Theorem 1])

$$
d\left(b^{n}, q^{n-1}\right) \leqslant \frac{1}{2^{2 n-1}}|V|
$$

Applying the same method as in Farin's case, we obtain the coefficients of the approximation by

$$
q_{i}=\frac{(-1)^{i}}{\left(\begin{array}{c}
n-1 \\
i
\end{array}\right)}\left\{\sum_{j=0}^{i}(-1)^{j}\left(\begin{array}{c}
n \\
j
\end{array}\right) b_{j}-\lambda_{i} V\right\}
$$

If we artificially elevate the degree of $q^{n-1}$, then

$$
q^{n-1}(t)=\sum_{i=0}^{n}\left\{b_{i}-\left(\lambda_{i}-\lambda_{i-1}\right) \frac{(-1)^{i}}{\left(\begin{array}{l}
n \\
i
\end{array}\right)} V\right\} B_{i}^{n}(t)
$$

From these, we have

$$
\begin{aligned}
\alpha_{i} & =\lambda_{i}-\lambda_{i-1} \\
& =\frac{1}{2^{2 n-1}}\left(\begin{array}{c}
2 n \\
2 i
\end{array}\right)
\end{aligned}
$$

and all the $\beta_{i}$ are zero, and also the error bound is given by (4.1). 


\section{REFERENCES}

[1] W.L.F. Degen, 'Best approximation of parametric curve by splines', in Mathematical methods in computer aided design II, (T. Lyche and L. L. Schumaker, Editors) (Academic Press, New York, 1992).

[2] M. Eck, 'Degree reduction of Bézier curves', Comput. Aided Geom. Design 10 (1993), 237-251.

[3] E. Eisele, 'Chebyshev approximation of planar curves by splines', J. Approx. Theory 76 (1994), 133-148.

[4] G. Farin, 'Algorithms for rational Bézier curves', Comput. Aided Design 15 (1983), 73-77.

[5] G. Farin, Curves and surfaces for computer aided geometric design (Academic Press, New York, 1988).

[6] A.R. Forrest, 'Interactive interpolation and approximation by Bézier polynomials', Comput. Aided Design 22 (1990), 527-537.

[7] M.A. Lachance, 'Chebyshev economization for parametric surfaces', Comput. Aided Geom. Design 5 (1988), 195-208.

[8] Y. Park and U.J. Choi, 'Degree reduction of Bézier curves and its error analysis', $J$. Austral. Math. Soc., Ser. B (to appear).

[9] M.A. Watkins and J.A. Worsey, 'Degree reduction of Bézier curves', Comput. Aided Des. 20 (1988), 398-405.

Departmentof Mathematics

Korea Advanced Institute of

Science and Technology

Gu-sung Dong, Yu-sung Gu

Taejon 305-701

Republic of Korea

Department of Computer Science

A-Jou University

Suwon

Republic ofKorea
Department of Mathematics

Korea Advanced Institute of Science and Technology

Gu-sung Dong, Yu-sung Gu

Taejon 305-701

Republic of Korea 